\title{
Identification and characterization of expressed retrotransposons in the genome of the Paracoccidioides species complex
}

Marco Aurélio Soares ${ }^{1 \dagger}$, Roberta Amália de Carvalho Araújo ${ }^{1 \dagger}$, Marjorie Mendes Marini ${ }^{3}$, Luciana Márcia de Oliveira ${ }^{2,6}$, Leonardo Gomes de Limaํ, Viviane de Souza Alves', Maria Sueli Soares Felipe ${ }^{4}$, Marcelo Macedo Brigido ${ }^{4}$, Celia Maria de Almeida Soares ${ }^{5}$, Jose Franco da Silveira ${ }^{3}$, Jeronimo Conceição Ruiz ${ }^{6}$ and Patrícia Silva Cisalpino ${ }^{1,2^{*}}$

\begin{abstract}
Background: Species from the Paracoccidioides complex are thermally dimorphic fungi and the causative agents of paracoccidioidomycosis, a deep fungal infection that is the most prevalent systemic mycosis in Latin America and represents the most important cause of death in immunocompetent individuals with systemic mycosis in Brazil. We previously described the identification of eight new families of DNA transposons in Paracoccidioides genomes. In this work, we aimed to identify potentially active retrotransposons in Paracoccidioides genomes.

Results: We identified five different retrotransposon families (four LTR-like and one LINE-like element) in the genomes of three Paracoccidioides isolates. Retrotransposons were present in all of the genomes analyzed. P. brasiliensis and P. lutzii species harbored the same retrotransposon lineages but differed in their copy numbers. In the Pb01, Pb03 and $\mathrm{Pb} 18$ genomes, the number of LTR retrotransposons was higher than the number of LINE-like elements, and the LINE-like element RtPc5 was transcribed in Paracoccidioides lutzii (Pb01) but could not be detected in P. brasiliensis (Pb03 and Pb18) by semi-quantitative RT-PCR.

Conclusion: Five new potentially active retrotransposons have been identified in the genomic assemblies of the Paracoccidioides species complex using a combined computational and experimental approach. The distribution across the two known species, $P$. brasiliensis and $P$. lutzii, and phylogenetics analysis indicate that these elements could have been acquired before speciation occurred. The presence of active retrotransposons in the genome may have implications regarding the evolution and genetic diversification of the Paracoccidioides genus.
\end{abstract}

\section{Background}

Transposable elements (TEs) have been found in virtually all eukaryotic species investigated to date $[1,2]$ and may represent a significant portion of the genomes of living organisms. TEs can account for $80 \%$ or more of total genomic DNA in plants and comprise $45 \%$ and $20 \%$ of the genomes of metazoans and fungi, respectively

\footnotetext{
* Correspondence: pscisalp@icb.ufmg.br

${ }^{\dagger}$ Equal contributors

'Departamento de Microbiologia, Instituto de Ciências Biológicas, Universidade Federal de Minas Gerais, 31270-901 Belo Horizonte, MG, Brazil

${ }^{2}$ Programa de Pós-graduação em Bioinformática, Instituto de Ciências Biológicas, Universidade Federal de Minas Gerais, 31270-901 Belo Horizonte, MG, Brazil

Full list of author information is available at the end of the article
}

$[3,4]$. TEs are DNA sequences with the ability to move from one genomic location to another and can be grouped in two classes according to whether their transposition intermediate is RNA (class I or retrotransposons) or DNA (class II or DNA transposons) [2,5].

Retrotransposons replicate by a "copy and paste" process, whereby the RNA intermediate is reverse-transcribed into double-stranded (ds) DNA by enzymes encoded by the TEs themselves. Elements belonging to class I are further divided into five orders based on their mechanistic features, organization and reverse transcriptase phylogeny: LTR retrotransposons, DIRS-like elements, Penelope-like elements, LINEs and SINEs [2,6]. LTR retrotransposons are the most widespread, especially those from the Gypsy and Copia superfamilies. Members of the LTR order usually encode 
two open reading frames (ORFs), one related to viral structural proteins ( $\mathrm{gag}$ ) and the second, known as pol, to a polyprotein composed of an aspartic protease (AP), a reverse transcriptase (RT), an RNase $\mathrm{H}(\mathrm{RH})$ and an integrase (IN) $[2,6]$.

Although fungal genomes generally contain fewer repetitive sequences than higher eukaryotes, TEs are viewed as central agents in the evolution of fungal genomes [2,3,7]. In Magnaporthe oryzae, clusters of TEs were associated with increased rates of chromosomal rearrangements, gene duplication and gene evolution [8]. There is an apparent correlation between TE clustering and chromosomal polymorphism in Fusarium oxysporum [9], and in Aspergillus niger, recombination mediated by retrotransposons has led to inversions of genomic regions [10]. In Verticillium dahliae, an asexual plant pathogen, chromosomal rearrangements were found to be associated with retrotransposons, and the authors suggest that homologous recombination between highly similar copies of transposable elements might help to generate genetic diversity [11].

Paracoccidioides is a thermally dimorphic fungus that infects approximately 10 million people in Latin America, causing paracoccidioidomycosis, the most prevalent systemic fungal disease in this region and the deep mycosis responsible for the most deaths in immunocompetent individuals in Brazil $[12,13]$. Until 2006, the genus Paracoccidioides was believed to include only one species: Paracoccidioides brasiliensis [14]. It was only through Multilocus Sequence Typing (MLST) analysis that the genetic variability in this genus, formerly believed to be merely intraspecific and due to geographic polymorphism, was revealed. Four cryptic species, S1, PS2 and PS3, were identified from the $P$. brasiliensis complex $[15,16]$, as well as the new species Paracoccidioides lutzii (originally called Pb01-like) $[17,18]$.

Since 2009, it has been accepted that the Paracoccidioides genus is composed of four distinct phylogenetic lineages (S1,PS2, PS3 and Pb01-like), which vary in their virulence, culture adaptation and the different host immune responses they induce $[15,19,20]$. Strain $\mathrm{Pb} 18$ is a member of Species 1 (S1), which is composed of 38 isolates among the 65 studied and is distributed across Latin America [15]. The Pb03 isolate belongs to phylogenetic species 2 (PS2), which is composed of one Venezuelan and five Brazilian isolates among the 65 studied. The extensively studied clinical isolate $\mathrm{Pb} 01$ (Paracoccidioides lutzii) is phylogenetically distinct from the other strains [17].

A comparative genomic analysis of three Paracoccidioides species-two P. brasiliensis ( $\mathrm{Pb} 03$ and $\mathrm{Pb} 18$ ) and one P. lutzii (Pb01)-identified all types of TEs. TEs correspond to approximately $8-9 \%$ of $P$. brasiliensis genomes and $16 \%$ of the $P$. lutzii genome [21]. We previously described a systematic survey of sequenced Paracoccidioides genomes for class II TEs (transposons) that resulted in the identification of eight families of DNA transposons. A detailed analysis of class II elements belonging to the Tc1/mariner superfamily revealed an unequal distribution among Paracoccidioides species and raised the possibility of the presence of active elements in the fungus genome [22]. TE insertions near genes can modify gene expression patterns, while insertions within genes can interrupt transcription and gene function [23]. In this work, we aimed to identify potentially active retrotransposons in the Paracoccidioides genome using a Paracoccidioides EST database to search for retrotransposons with evidence of transcription.

We expanded our search for class I TEs (retrotransposons) to identify and characterize potentially active retrotransposons in the Paracoccidioides species complex. We identified five different retrotransposons (four LTR-like and one LINE-like element) in the genomes of the three isolates. Whereas some DNA transposons are unequally distributed in the genomes of the Paracoccidioides species complex, retrotransposons are present in all of the genomes analyzed.

\section{Results}

\section{Clustering, similarity and functional annotation of Paracoccidioides EST sequences}

As shown in the workflow in Figure 1, the first step in the identification of active retrotransposons in the genomes of the Paracoccidioides species complex was to access the information contained in the EST database (http://www. ncbi.nlm.nih.gov/genbank/dbest/). A local database was built with 41,558 downloaded Paracoccidioides ESTs, which were then clustered, resulting in 12,922 sequence clusters distributed in 4,812 contigs and 8,110 singlets. The EST clusters were compared with sequences deposited in three databases: NR (the NCBI non-redundant protein database), TEfam and Repbase (Figure 1). Approximately $20 \%$ of the clusters $(2,544 / 12,922)$ showed no similarity with any sequences in the NR database with the parameters set (see Methods section); Blast hit descriptions were analyzed based on a lexical search approach using specific keywords resulting in a first set of 142 EST sequences of putative retrotransposons (Figure 1). Further searches against specific databases identified 809 EST sequences common to the TEfam and Repbase databases $(6.2 \%$ of all clusters) (Figure 1). A set of 52 EST sequences that were found to be common between the results from similarity and lexical searches were mapped in the Paracoccidioides genomes.

\section{Identification and characterization of Paracoccidioides retrotransposons}

Employing the set of 52 EST clusters as queries against the Paracoccidioides genomes, five genomic sequences 


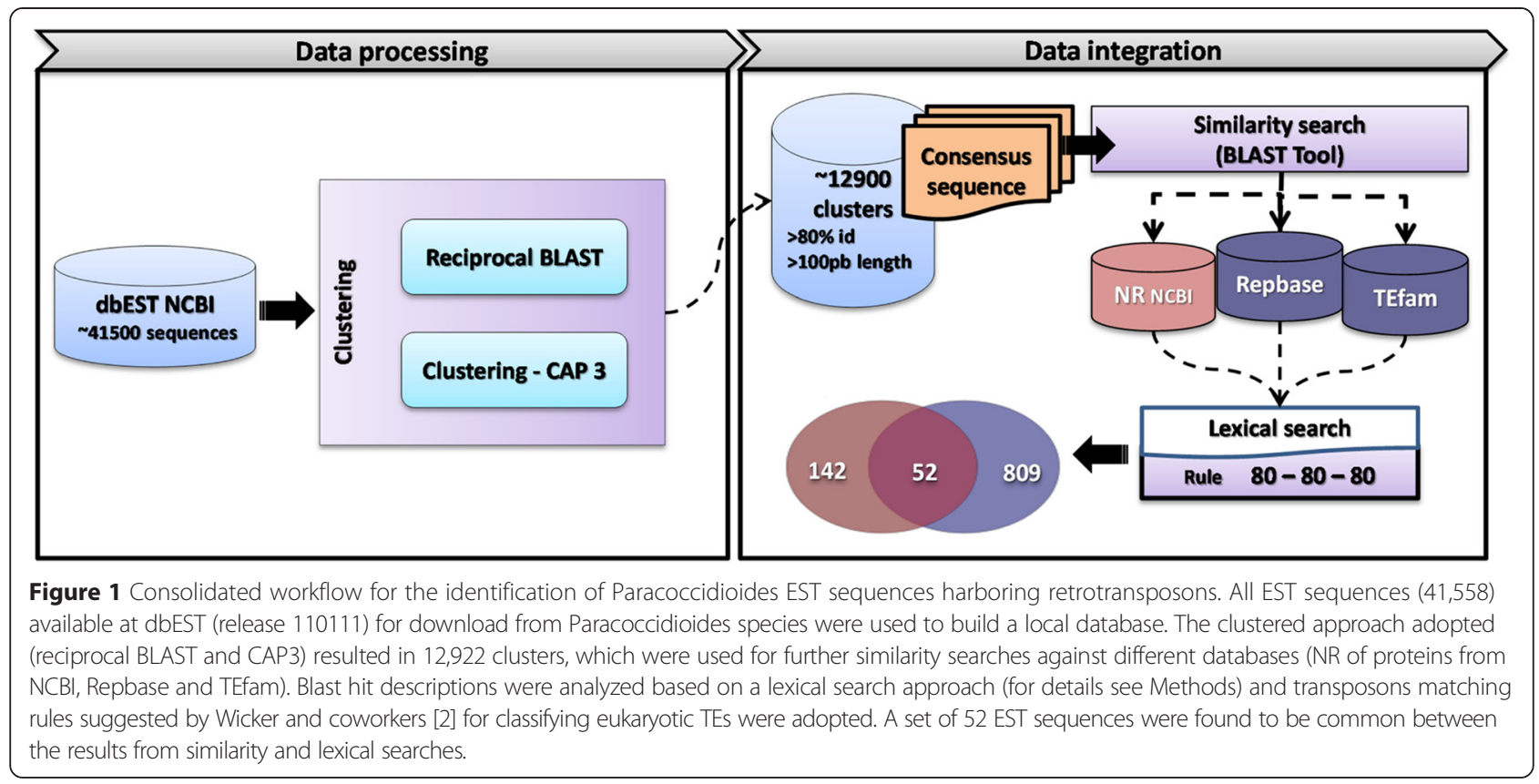

with characteristics of retrotransposons were identified and are referred to henceforth as RtPc (Retrotransposon Paracoccidioides complex) 1 to 5 (Table 1). All RtPc elements showed similarity with retrotransposons (Class I) (Table 1). Twenty-seven EST clusters were anchored to these five genomic sequences, each corresponding to a putative retrotransposon, distributed as follows: 18 clusters mapped to RtPc1, three to RtPc3 and RtPc5, two to RtPc4 and only one to RtPc2 (Additional file 1). The remaining 25 EST groups were mapped to genomic sequences with a large number of stop codons, preventing identification of complete copies of retrotransposons.

Similarity searches were conducted to locate and perform the functional and structural annotation of intact, full copy elements in all supercontigs of $P$. brasiliensis (isolates $\mathrm{Pb} 18$ and $\mathrm{Pb} 03$ ) and P. lutzii (isolate $\mathrm{Pb} 01$ ), the three Paracoccidioides genomes (http://www.broadinstitute.org) [21]. Consensus sequences were generated by alignment of all intact copies of each retrotransposon (Additional file 2). We found that there is at least one intact copy of each RtPc element in one or more sequenced Paracoccidioides genome. The elements were classified (Table 1) based on the results of alignments with sequences of retrotransposons from the GIRI database (http://www.girinst.org/ censor/index.php) using the criteria proposed by Wicker et al. [2] and Kapitonov et al. [24] (http://www.girinst.org/ RTphylogeny/RTclass1/) [24]. We identified four RtPc elements belonging to the LTR order from the Gypsy (LTRGypsy-RtPc1 and LTR-Gypsy-RtPc2) and Copia (LTRCopia-RtPc3 and LTR-Copia-RtPc4) superfamilies, and one element is a LINE retrotransposon (LINE-Tad-likeRtPc5) (Table 1, Figure 2) that we initially identified as a new type of fungal non-LTR retrotransposon related to the Tad clade.

\section{LTR retrotransposons of the superfamily Gypsy (LTR- Gypsy-RtPc)}

The Gypsy elements identified in Paracoccidioides complex were 5412 to $5740 \mathrm{bp}$ in length, with the coding regions flanked by LTRs. Primer binding sites PBS and

Table 1 Classification of retrotransposons identified in Paracoccidioides genomes

\begin{tabular}{|c|c|c|c|c|c|}
\hline \multicolumn{4}{|c|}{ Classification } & \multicolumn{2}{|l|}{ Identity of the best hit } \\
\hline Element & Class & Order & Superfamily/clade & Organism & $\%$ Similarity \\
\hline RtPc1 & I (Retrotransposons) & LTR & Gypsy & Aspergillus fumigatus & 64.9 \\
\hline RtPc2 & I (Retrotransposons) & LTR & Gypsy & Aspergillus nidulans & 64.4 \\
\hline RtPc3 & I (Retrotransposons) & LTR & Copia & Drosophila bipectinata & 68.6 \\
\hline RtPc4 & I (Retrotransposons) & LTR & Copia & Coccidioides posadasii & 62.6 \\
\hline RtPc5 & I (Retrotransposons) & LINE & // Tad-like* & Blumeria graminis & 63.9 \\
\hline
\end{tabular}

*as mentioned in the Results, element LINE-Tad-like_RtPc5 was identical to the non-LTR retrotransposon of the Tad-CgT family, which was previously identified as element PbNLR1 by Novikova et al. [25]. 


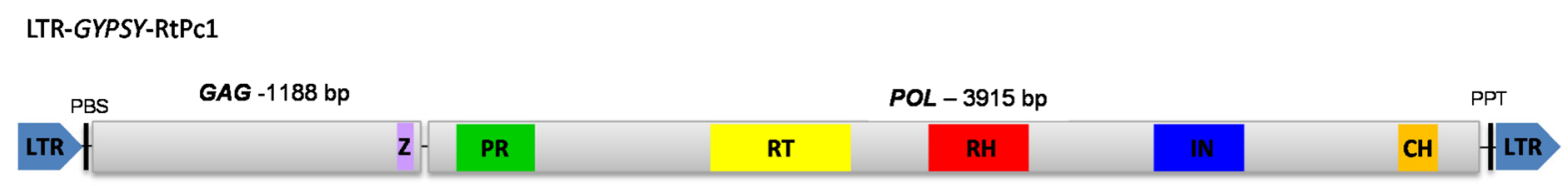

LTR-GYPSY-RtPc2

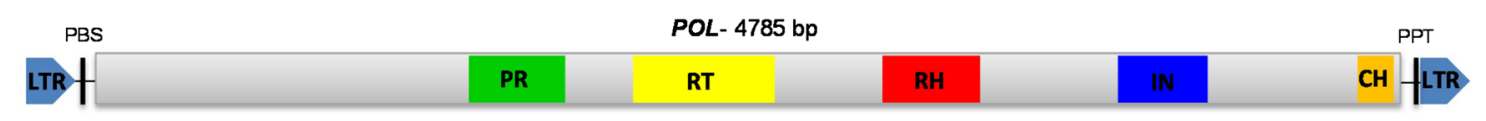

LTR-COPIA-RTPC3

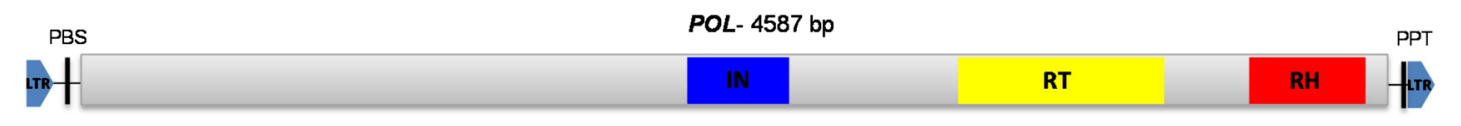

LTR-COPIA-RtPC4

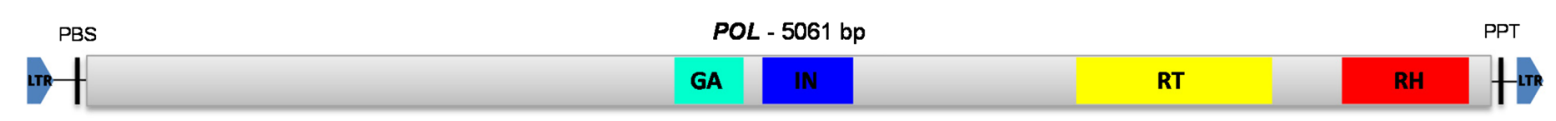

LINE-Tad-RtPc5

ORF -1716 bp

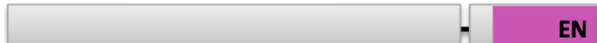

POL - 3939 bp

\section{RT}

Figure 2 Structure and organization of RtPc elements in the Paracoccidioides complex. Schematic representations of complete RtPc elements are shown. The LTRs are represented by blue arrowheads and PBS/PPT by black bars. The domains are represented as follows: zinc finger - light purple; protease (PR) - green; reverse transcriptase (RT) - yellow; RNase H (RH) - red; integrase (IN) - blue; chromodomain (CH) - orange; and endonuclease (EN) - pink. The ORF with its respective size is represented above each element. Figures are not to scale.

PPT were also identified at the $5^{\prime}$ and $3^{\prime}$ ends of each element, respectively (Additional file 3, Figure 2). The element LTR-Gypsy-RtPc1 (5740 bp) has two ORFs corresponding to the gag gene, which is predicted to encode the proteins of the virus-like particle (VLP), and the pol gene, which encodes a polyprotein that gives rise to RNase $\mathrm{H}$-reverse transcriptase, integrase and protease after processing. The Pol ORF was in a -1 frameshift in relation to the gag ORF. Coding sequences were flanked by 246-bp LTRs, with the nucleotides TG (initial) and CA (final). The first ORF (gag gene) encodes a 396-amino-acid (aa) protein with a conserved zinc finger domain (17 aa), and the second encodes a polyprotein that displays the following conserved domains: protease (99 aa), reverse transcriptase (176 aa), RNase H (126 aa), integrase (114 aa) and a chromodomain (52 aa). Full copies of LTR-Gypsy-RtPc2 (5412 bp) showed a single ORF that encodes a polyprotein with POL domains consisting of protease (96 aa), reverse transcriptase (178 aa), RNase $\mathrm{H}$ (123 aa) and integrase (113 aa). LTR-Gypsy-RtPc1 and LTR-Gypsy-RtPc2 shared $64.9 \%$ and $64.4 \%$ nucleotide sequence similarity, respectively, with retroelements identified in the fungus Aspergillus nidulans (Table 1).

\section{LTR retrotransposons from the Copia (LTR-Copia-RtPc) superfamily}

The Copia elements identified in the Paracoccidioides complex were 5181 to 5685 bp long with the coding regions flanked by LTRs. The primer binding sites PBS and PPT were also identified at the $5^{\prime}$ and $3^{\prime}$ ends of each element, respectively (Additional file 3, Figure 2). Comparison with conserved domain databases showed that the element LTRCopia-RtPc3 has a single ORF that encodes a 1528-aa protein with three conserved domains: integrase (126 aa), reverse transcriptase (253 aa) and RNase $H$ (143 aa). The LTR-Copia-RtPc4 element had a single ORF with fused gag and pol sequences predicted to encode a 606-aa polyprotein with the following domains: Gag (87 aa), integrase (114 aa), reverse transcriptase (245 aa) and RNase H (160 aa). The elements LTR-Copia- RtPc3 and RtPc4 shared 68.6 and $62.6 \%$ nucleotide sequence similarity with retrotransposons 
identified in Drosophila bipectinata and in the fungus Coccidioides posadasii, respectively (Table 1).

\section{A Non-LTR retrotransposon similar to the Tad clade elements (LINE-Tad-RtPc5)}

The complete element LINE-Tad-like-RtPc5 (5905 bp) contains two separate ORFs: the first encodes a protein (572 aa) with no similarity to any known protein, and the second, with a frameshift in relation to the first one, encodes a protein (1312 aa) that has conserved endonuclease (222 aa), reverse transcriptase (267 aa) and RNase H (145 aa) domains. This element has no LTRs, but a region corresponding to a poly A tail was identified (Additional file 3, Figure 2). LINE-Tad-RtPc5 shared $63.9 \%$ nucleotide sequence similarity with a retrotransposon identified in the fungus Blumeria graminis (Table 1). To assign this element to a specific clade, an automated tool (RtClass1, http://www.girinst.org/RTphylogeny/RTclass1/) [24] was employed that uses phylogenetic analysis of the RT domain protein. Based on the results from the RTclass1 tool, our LINE element clustered together with the Tad1 clade, although the RtPc5 reverse transcriptase was indicated as belonging to an outgroup clade. In an attempt to find better information on non-LTR retrotransposons related to fungi, we uncovered an interesting report [25] in which the authors employed an in silico approach to survey the nonLTR retrotransposons in 57 fungal genomes, reporting more than 100 novel non-LTR retrotransposons and, importantly, describing two new clades, Inkcap and Deceiver. $P$. brasiliensis isolate Pb01 (now P. lutzii) was listed among the species searched by the authors, and three novel Tadlike elements, identified as PbNLR1, PbNLR2 and PbNLR3, were reported among the novel non-LTR elements harbored by this genome. Based on the phylogeny of the RT domains, the authors classified these novel P. brasiliensis non-LTR elements in the Tad clade under distinct families: CgT, Ask1 and Ask2. On the basis of sequence identity, it was possible to establish the identity of the LINE-Tad-RtPc5 described here with the PbNLR1 element cited by [25], which is a Tad-CgT element.

\section{Distribution of retrotransposons in the Paracoccidioides species complex}

After identifying at least one complete copy of each of the five elements, the RtPc sequences were used to identify intact and truncated copies in the sequenced Paracoccidioides genomes (Table 2). Overall, 538 copies of RtPc elements were found scattered throughout the genomes of the three isolates. The majority of RtPc elements (54.46\%) were identified in P. lutzii ( $\mathrm{Pb01})$, and the remaining 245 copies were found in P. brasiliensis isolates (17.28\% and $28.26 \%$ in $\mathrm{Pb} 03$ and $\mathrm{Pb} 018$, respectively) (Table 2). The distribution of copies of each element in the sequence supercontigs is shown in Additional file 4. Out of 538 retroelements identified in the Paracoccidioides genomes, 514 (95.54\%) were truncated and only 24 (4.46\%) were intact (Table 2). The distribution of truncated forms in Paracoccidioides species was as follows: 284 copies in P. lutzii (Pb01) and the remaining copies in P. brasiliensis isolates (141 copies in Pb18 and 89 in $\mathrm{Pb} 03$ ) (Table 2). Most of the intact elements were found in the Pb18 P. brasiliensis isolate (11/24) and Pb01 P. lutzii isolate $(9 / 24)$, and four copies were found in the Pb03 $P$. brasiliensis isolate (Table 2). Gypsy elements were the most abundant retrotransposons in Paracoccidioides genomes, comprising approximately $53.34 \%$ (287/538) of the total retroelements identified in these species. Out of 111 copies of LTR-Gypsy-RtPc1, 57 were found in P. lutzii, followed by 39 and 15 in isolates $\mathrm{Pb} 18$ and $\mathrm{Pb} 03$, respectively. Four intact copies of RtPc1 were present in P. lutzii, and eight were present in Pb18 (Table 2). The LTR-Gypsy-RtPc2 element was the most abundant RtPc element (176/538) of all the retrotransposons identified in the Paracoccidioides genomes studied here. It is interesting to note that $56.81 \%$ of LTRGypsy-RtPc2 copies (100/172) were found in P. lutzii (isolate $\mathrm{Pb} 01$ ) and that all of these were truncated. Only one intact copy of this element was found in the genomes of $P$. brasiliensis isolates (Table 2).

Copia and LINE retrotransposons comprised $22.5 \%$ and $24.16 \%$ of all the retroelements in the Paracoccidioides genomes, respectively. Out of eighteen LTR-Copia-RtPc3 copies, ten were found in the isolate $\mathrm{Pb} 03$, followed by five in $\mathrm{Pb} 01$ and three in Pb18. As for the LTR-Copia-RtPc4 element, most copies were found in P. lutzii $(\mathrm{n}=66)$, followed by $P$. brasiliensis isolates $\mathrm{Pb} 03(\mathrm{n}=28)$ and $\mathrm{Pb} 18(\mathrm{n}=9)$. For the LINE-Tad-RtPc5 element, again, most copies $(\mathrm{n}=65)$ were identified in P. lutzii (Pb01), followed by isolates $\mathrm{Pb} 03(\mathrm{n}=34)$ and Pb18 (31). Most of these elements were truncated in the isolate $\mathrm{Pb} 01$ (92.3\%) (Table 2).

\section{LTRs not associated with complete retrotransposons}

In addition to intact and truncated elements, structural variations of LTR retrotransposons include solo LTRs, which together with LTR remnants are believed to be the result of unequal recombination and illegitimate recombination. We identified 468 copies of solo LTRs closely related to Gypsy- and Copia-RtPc elements; most of these were found in P. lutzii $(\mathrm{n}=222)$, followed by $P$. brasiliensis isolates Pb18 $(\mathrm{n}=164)$ and Pb03 $(\mathrm{n}=81)$ (Additional file 4). Solo LTRs belonging to the Gypsy superfamily are far more abundant (2.6-fold) than those of Copia-like retrotransposons. The ratio of solo LTR sequences to intact elements was 13.4 in Paracoccidioides genomes, and the ratio of solo-Gypsy LTRs to intact elements was also higher than that for solo-Copia LTRs vs. intact elements (Additional file 4). 
Table 2 Distribution of retrotransposons in Paracoccidioides species genomes

\begin{tabular}{|c|c|c|c|c|c|c|c|c|c|}
\hline \multirow{4}{*}{ Elements } & \multicolumn{3}{|c|}{ Isolate $\mathrm{Pb} 01$} & \multicolumn{3}{|c|}{ Isolate Pb03 } & \multicolumn{3}{|c|}{ Isolate $\mathrm{Pb} 18$} \\
\hline & \multicolumn{3}{|c|}{ (P. lutzii) } & \multicolumn{3}{|c|}{ (P. brasiliensis) } & \multicolumn{3}{|c|}{ (P. brasiliensis) } \\
\hline & Copy & Intact & Truncated & Copy & Intact & Truncated & Copy & Intact & Truncated \\
\hline & \multicolumn{3}{|c|}{ Number } & \multicolumn{3}{|c|}{ Number } & \multicolumn{3}{|c|}{ Number } \\
\hline LTR-Gypsy-RtPc1 & 57 & 4 & 53 & 15 & 0 & 15 & 39 & 8 & 31 \\
\hline LTR-Gypsy-RtPc2 & 100 & 0 & 100 & 6 & 0 & 6 & 70 & 1 & 69 \\
\hline LTR-Copia-RtPc3 & 5 & 0 & 5 & 10 & 3 & 7 & 3 & 1 & 2 \\
\hline LTR-Copia-RtPc4 & 66 & 0 & 66 & 28 & 0 & 28 & 9 & 1 & 8 \\
\hline LINE-Tad-CgT-RtPc5 & 65 & 5 & 60 & 34 & 1 & 33 & 31 & 0 & 31 \\
\hline Subtotal & 293 & 9 & 284 & 93 & 4 & 89 & 152 & 11 & 141 \\
\hline
\end{tabular}

The presence of RtPc elements in Paracoccidioides isolates of distinct phylogenetic origins

To investigate the occurrence of RtPc elements, a segment of the coding sequence for reverse transcriptase was PCR amplified from the genomic DNA of 31 isolates, including Pb01-like $P$. lutzii isolates and isolates belonging to the Paracoccidioides phylogenetic lineages S1, PS2 and PS3 from the P. brasiliensis complex (Table 3, Figure 3). Reverse transcriptase was present in 24 isolates. The identity of the amplicons was confirmed by sequencing a 300-bp fragment corresponding to the coding region for the reverse transcriptase of each of the five elements (isolates $\mathrm{Pb} 01, \mathrm{~Pb} 03$ and $\mathrm{Pb} 18$ ) (data not shown). The element LINE-Tad-RtPc5 was present in all isolates. No Gypsy element was found in isolates EPM81 and EPM102. No correlation was found between the distribution patterns of RtPc elements and the phylogeny of Paracoccidioides lineages.

Genomic organization and transcription of RtPc elements We also analyzed the genomic organization of RtPc elements by Southern blot hybridization using probes corresponding to the retrotransposons LTR Gypsy-RtPc1 and LINE-Tad-RtPc5. Figure 4 shows the results obtained using genomic DNA from $P$. lutzii (Pb01) and P. brasiliensis (Pb18). As expected from the in silico analysis, the LTR-Gypsy-RtPc1 probe hybridized to multiple genomic fragments from P. lutzii (Pb01) and P. brasiliensis (Pb18), confirming the polymorphic nature and abundance of this element. The number and signal intensity of hybridizing fragments identified in $\mathrm{Pb} 01$ was higher than in $\mathrm{Pb} 18$, confirming the variation in the copy number of LTRGypsy-RtPc1. The hybridization patterns obtained with the element LINE-Tad-RtPc5 indicate that these elements were more abundant in the $P$. lutzii genome.

LTR-Gypsy-RtPc1 and LINE-Tad-RtPc5 elements were mapped to the chromosomal bands of isolates $\mathrm{Pb} 01$ and Pb18 (Figure 4, panels C and F), which had been separated by PFGE. Pb01 and Pb18 showed distinct karyotype profiles with four and five chromosomal bands, respectively.
Isolate B339 was used as a reference for chromosomal band size. The LTR-Gypsy-RtPc1 probe hybridized to three and four chromosomal bands in isolates $\mathrm{Pb} 01$ and $\mathrm{Pb} 18$, respectively (Figure 4, panel C). The LINE-Tad-RtPc5 probe hybridized to three and two chromosomal bands in isolates $\mathrm{Pb} 18$ and $\mathrm{Pb} 01$, respectively (Figure 4, panel F).

To detect transcripts of RtPc elements, semi-quantitative RT-PCR was performed using cDNAs from isolates Pb01 (P. lutzii), Pb03 and Pb18 (P. brasiliensis, PS2 and S1). Figure 5 shows that the five retrotransposons were transcribed in the yeast form of $P$. lutzii ( $\mathrm{Pb01})$, but only RtPc1, RtPc2, RtPc3 and RtPc4 were transcribed in the yeast form of P. brasiliensis (Pb03 and Pb18).

\section{Phylogenetic analysis of RtPc elements}

The reverse transcriptase domains from the 24 complete elements were employed to establish the phylogenetic relationship between RtPc elements derived from each of the two species of the genus Paracoccidioides, P. brasiliensis (isolates $\mathrm{Pb} 18$ and $\mathrm{Pb} 03$ ) and $\mathrm{P}$. lutzii (isolate $\mathrm{Pb} 01$ ). Intact copies of LTR-Gypsy-RtPc1 and LINE-Tad-RtPc5 have been identified in P. brasiliensis and P. lutzii. In the phylogenetic tree (Figure 6A), three major clusters can be distinguished. One group contains the LTR-Gypsy elements, the second contains LTR-Copia elements and the third contains the LINE elements, all strongly supported by high posterior probabilities, thus confirming the classification of these elements. The phylogenetic tree for the LTR-Gypsy-RtPc1 element (Figure 6B) showed 3 clusters, two of which were composed of species-specific sequences from $P$. brasiliensis (Pb18) and P. lutzii (Pb01). The first branch consisted of sequences exclusively from $P$. brasiliensis (Pb18), comprising 6 of the 8 intact copies found in this species (supercontigs $1.4,1.6,1.7,1.10,1.11$ and 1.14); the central branch was composed of two RtPc sequences from $P$. brasiliensis (Pb18, supercontigs 1.1 and 1.3) and three from P. lutzii (Pb01, supercontigs 1:22, 1:19, 1:29 and 1:16), and a high similarity was observed among the five elements inside this cluster (Figure 6B). The grouping of elements from these two different species illustrates the degree of similarity 
Table 3 Paracoccidioides isolates used in this study

\begin{tabular}{|c|c|c|c|}
\hline Isolate & Origin & Country & $\begin{array}{l}\text { Phylogenetic } \\
\text { species }^{c}\end{array}$ \\
\hline$\overline{\mathrm{PbO}^{\text {af }}}$ & clinical & Brazil - Goiás & P. lutzii \\
\hline $\mathrm{Ed} 01^{\text {ef }}$ & clinical & Brazil - Goiás & P. lutzii \\
\hline $1578^{\text {ef }}$ & clinical & Brazil - Goiás & P. lutzii \\
\hline Pb03 ${ }^{\text {adeg }}$ & chronic PCM ${ }^{b}$ & Brazil - São Paulo & P. brasiliensis - PS2 \\
\hline $\mathrm{Pb} 4^{\text {deg }}$ & chronic PCM & Brazil - São Paulo & P. brasiliensis - PS2 \\
\hline $\mathrm{Pb} 2^{\text {deg }}$ & chronic PCM & Venezuela & P. brasiliensis - PS2 \\
\hline EPM77 & clinical & Colombia & P. brasiliensis - PS3 \\
\hline EPM $83^{e}$ & chronic PCM & Colombia & P. brasiliensis - PS3 \\
\hline Pb18 ade & chronic PCM & Brazil - São Paulo & P. brasiliensis - S1 \\
\hline $\mathrm{Pb} 5^{\mathrm{g}}$ & chronic PCM & Brazil - Paraná & P. brasiliensis - S1 \\
\hline B339 deg & chronic PCM & Brazil - São Paulo & P. brasiliensis - S1 \\
\hline $\mathrm{Pb}^{\text {deg }}$ & chronic PCM & Brazil - Paraná & P. brasiliensis - S1 \\
\hline $\mathrm{Pb} 11^{\text {deg }}$ & acute PCM & Brazil - Paraná & P. brasiliensis - S1 \\
\hline $\mathrm{Pb}^{\mathrm{d}}$ & clinical & Venezuela & P. brasiliensis - S1 \\
\hline $\mathrm{Pb} 10^{\text {deg }}$ & acute PCM & Peru & P. brasiliensis - S1 \\
\hline Penguin de & penguin feces & Uruguay & P. brasiliensis - S1 \\
\hline Utero $^{f}$ & chronic PCM & Argentina & P. brasiliensis - S1 \\
\hline $\mathrm{Pb} 13^{\text {deg }}$ & acute PCM & Brazil - Goiás & P. brasiliensis - S1 \\
\hline $\mathrm{Pb} 14^{\text {deg }}$ & acute PCM & Brazil - São Paulo & P. brasiliensis - S1 \\
\hline Pb9 deg & chronic PCM & Brazil - São Paulo & P. brasiliensis - S1 \\
\hline $63265^{e}$ & acute PCM & Argentina & P. brasiliensis - S1 \\
\hline $\mathrm{Pb}^{\mathrm{d}}$ & chronic PCM & Brazil - São Paulo & P. brasiliensis - S1 \\
\hline EPM59 & chronic PCM & Venezuela - Caracas & ND \\
\hline EPM62 & acute PCM & Venezuela - Caracas & ND \\
\hline EPM69 & chronic PCM & Venezuela - Caracas & ND \\
\hline EPM81 & clinical & Colombia - Medellin & ND \\
\hline EPM82 & chronic PCM & Colombia - Bogotá & ND \\
\hline EPM87 & acute PCM & Argentina - Entre Rios & ND \\
\hline EPM92 & Clinical & Brazil - Piauí & ND \\
\hline EPM97 & chronic PCM & Brazil - Maringá & ND \\
\hline EPM101 & Armadillo & Brazil -lbiá & ND \\
\hline EPM102 & Armadillo & Brazil - Ibiá & ND \\
\hline EPM117 & Armadillo & - & ND \\
\hline EPM141 & clinical & Brazil - Paraná & ND \\
\hline
\end{tabular}

${ }^{\text {a }} P$. brasiliensis isolates used in the genome sequencing project - Broad Institute of MIT and Harvard.

${ }^{b}$ PCM - paracoccidioidomycosis.

'Phylogenetic Species grouped in accordance with Matute et al. [15] ${ }^{\mathbf{d}}$, Teixeira et al. [17] ${ }^{\mathbf{e}}$ and Marini et al. [22] ${ }^{\mathbf{f}}$.

Isolate was also used by Morais et al. [59].

between these elements and suggests a common ancestry. The third branch harbored only one $P$. lutzii sequence (supercontig 1.16) (Figure 6B). This pattern suggests that the RtPc1 elements from P. brasiliensis and P. lut$z i i$ share sequences that were present in a relatively recent common ancestor, thus supporting the hypothesis of pre-speciation emergence of RtPc1 insertions. The RtPc3-Copia elements clustered in very close branches and showed a high sequence similarity, which could be explained by the low number of copies analyzed and by the fact that intact copies were only identified in $P$. brasiliensis lineages $\mathrm{Pb} 03$ and $\mathrm{Pb} 18$. However, for the LINE-Tad-RtPc5 element (Figure 6C), it was possible to observe a species-specific grouping, although only a single copy of RtPc5 has been analyzed in P. brasiliensis (isolate Pb03). Thus, despite the LINE-Tad-RtPc5 elements from P. brasiliensis and P. lutzii being located in separate branches, they nevertheless are phylogenetically close (Figure $6 \mathrm{C}$ ).

Based on the assumption that the two LTRs accumulate point mutations independently, the insertion time of an LTR retrotransposon could be estimated based on variations or sequence differences between the two LTRs ends, where would have been identical at the time of insertion [26]. Very low nucleotide divergence was observed in the LTRs of the 18 intact RtPc elements (Additional file 5). Considering that the analyzed elements are intact and most likely active, the estimated divergence between LTRs suggested recent insertion events in the genomes of P. brasiliensis and P. lutzii.

\section{Discussion}

Our group previously described a systematic survey of class II TEs (transposons) in the sequenced Paracoccidioides genomes that identified eight families of DNA transposons [22]. Here, we expand our search for class I TEs (retrotransposons) to identify and characterize potentially active retrotransposons in the Paracoccidioides species complex. To achieve this goal, a combined computational and experimental approach was applied to map these elements in the sequenced Paracoccidioides genomes (P. lutzii $-\mathrm{Pb} 01$ and P. brasiliensis - $\mathrm{Pb} 03$ and $\mathrm{Pb} 18)$. We identified five different retrotransposon families (four LTR-like and one LINElike element) in the genomes of the three isolates. Although some DNA transposons are unequally distributed in the three Paracoccidioides genomes, with some lineage-specific elements, retrotransposons are spread over all of the genomes analyzed. However, our data reveal several interesting characteristics of retrotransposon organization in Paracoccidioides species. For instance, $P$. brasiliensis and $P$. lutzii species harbor the same retrotransposon lineages but differ from one another in the number of copies present. $P$. lutzii contains 1.9 to 3.1 times more retrotransposons than P. brasiliensis isolates Pb18 and Pb03, respectively. Differences in LTR content were also observed between closely related species, such as those in the Coccidioides genus [27]. Another remarkable characteristic was the high number of truncated elements. For example, all Gypsy-RtPc2 and Copia-like insertions were degenerated in P. lutzii. Despite the differences in number and integrity between 


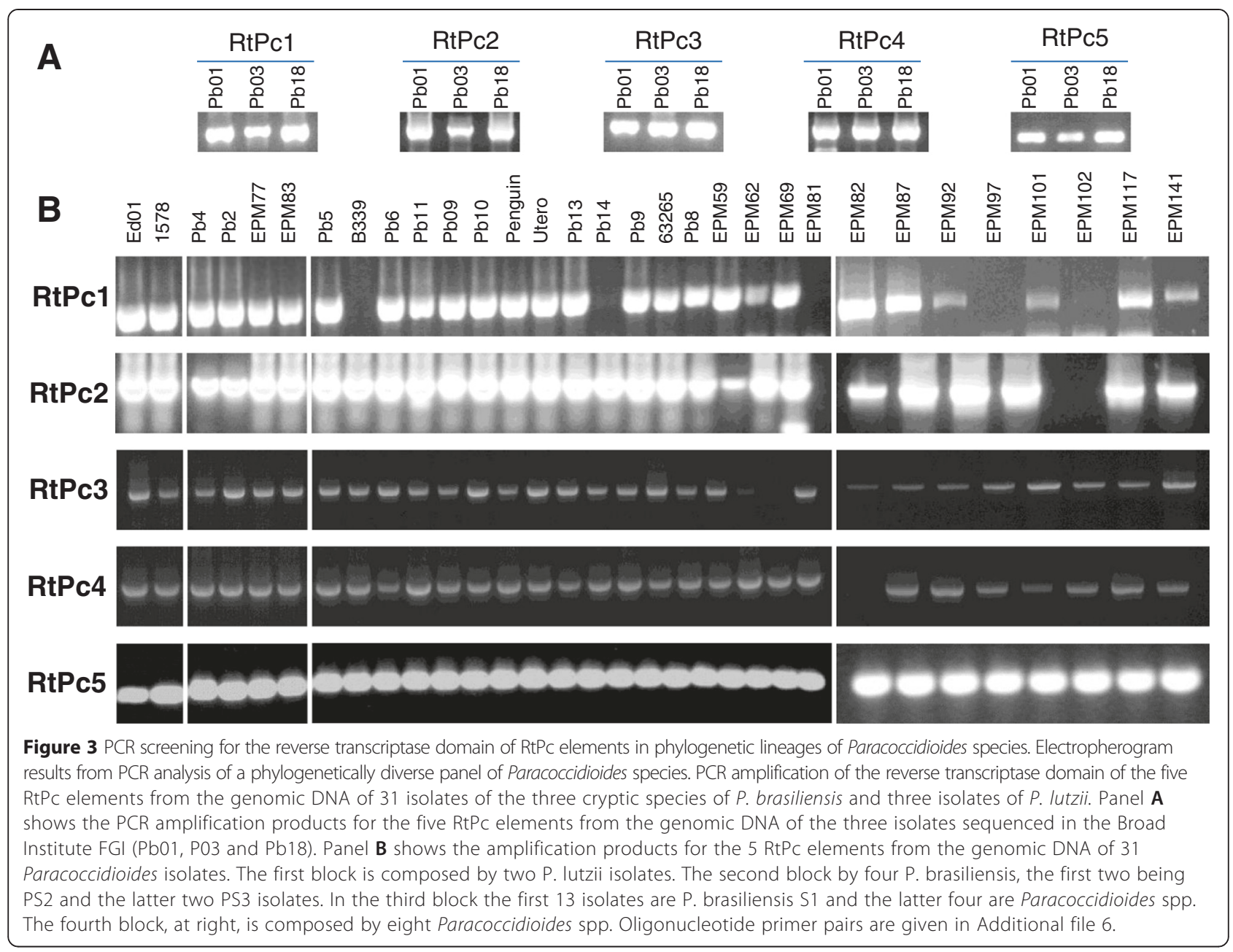

the five retrotransposon families, they were most likely acquired before the speciation event that gave rise to $P$. brasiliensis and P. lutzii, in contrast to some DNA transposons that appear to have been acquired after this event [22].

Desjardins et al. [21] performed a comparative genomic analysis and reported a high number of TEs in Paracoccidioides genomes. Mobile elements account for $16 \%$ of the $P$. lutzii genome and $7.7 \%$ and $9.2 \%$ of the genomes of $P$. brasiliensis isolates $\mathrm{Pb} 03$ and $\mathrm{Pb} 18$, respectively. A total of 971 retrotransposons were identified in the $P$. lutzii genome, a two-fold expansion compared with the $P$. brasiliensis genomes (338 and 460 elements in $\mathrm{Pb} 03$ and $\mathrm{Pb} 18$, respectively). In our work, we identified a smaller number of retrotransposons than previously described [21], with a total of 293 in Pb01, 93 in $\mathrm{Pb} 03$ and 152 in Pb18. This outcome may be explained by the fact that we searched for potentially active elements in an EST database, and given that highly defective elements are not represented in EST databases, they may not have been identified with this strategy.

LTR retrotransposons are classified into five superfamilies, of which Copia and Gypsy are found in fungal genomes, with Gypsy being the most abundant $[2,3,27]$. As expected for this type of genome, P. lutzii contains more Gypsy than Copia elements, and in isolate Pb18, there were nine times more of the former than of the latter. In isolate $\mathrm{Pb03}$, however, we identified 38 Copia and 21 Gypsy elements. In the global analysis of transposable elements performed by Desjardins et al. [21], slightly more Gypsy elements (85 copies) than Copia elements (80 copies) were found. In the three genomes they studied, there were more LTR retrotransposons than LINE-like elements.

Active transposable elements can be highly mutagenic, inserting into coding regions and leading to chromosomal breaks and rearrangements. Genomes tend to resist the expansion of TEs, and many organisms have developed genome defense systems that repress the activity of mobile elements [28-30]. Several mechanisms could be responsible for retrotransposon removal in Paracoccidioides genomes. One such mechanism could involve the erosion of LTR retrotransposons through the accumulation of point mutations or minor deletions. This accumulation could be due to repeat-induced point mutations (RIPs), as described in 


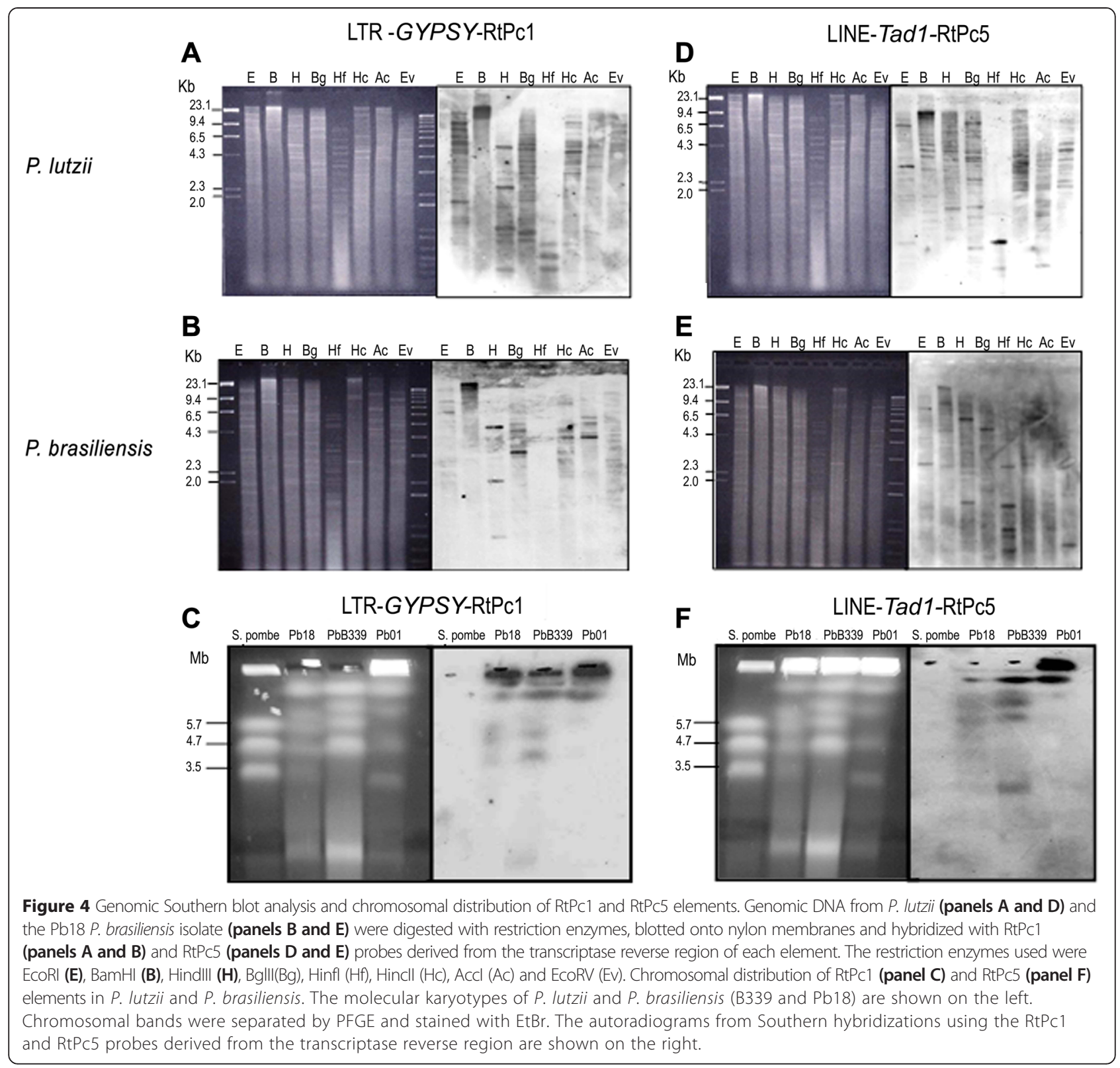

Neurospora [31], in which retrotransposons were inactivated by the accumulation of point mutations generated by an increased number of stop codons resulting from G-C to A-T transitions [27]. The presence of multiple stop codons was observed in the majority of retrotransposons identified in Paracoccidioides genomes in our study; in fact, only 5\% of the identified elements corresponded to intact copies. Analysis of GC content indicated an especially high AT content in LTR-Gypsy-RtPc2 and LTR-Copia-RtPc4 retrotransposons, which have the highest proportion of truncated elements. Desjardins et al. [21] also reported a lower CG content $(37 \%-39 \%)$ in transposon sequences than in the rest of the Paracoccidioides genome. The generation of solo-LTRs as a by-product of recombination between flanking LTRs of the same element could also be responsible for the deterioration of these elements. This seems to be the main mechanism driving the deterioration of Ty3/ Gypsy elements in Anopheles gambiae [32]. A large number of solo LTRs were found in all of the genomes analyzed in our study, indicating that a similar mechanism may have occurred in Paracoccidioides and could have played a role in the generation of the genomic plasticity observed in these fungi.

We used PCR to analyze the distribution of RtPc elements in 34 Paracoccidioides isolates of different geographic origin, four of which were environmental. Only LINE-Tad-RtPc5 was identified in all isolates, the elements LTR-Gypsy-RtPc2, LTR-Copia-RtPc3 and LTR- 


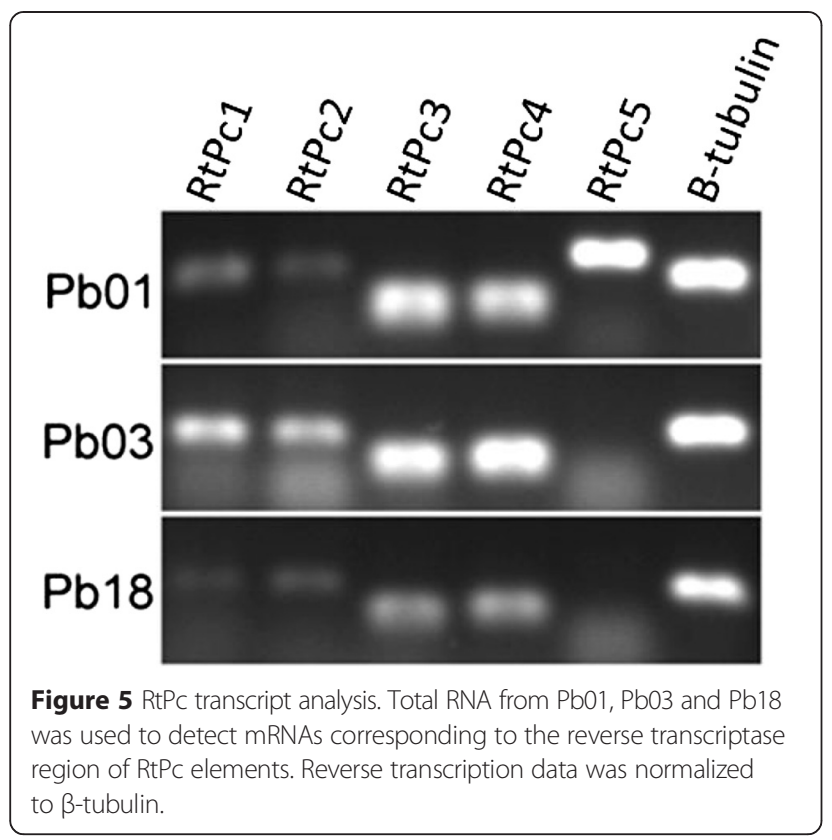

Copia-RtPc4 were absent in only one isolate, and the element LTR-Gypsy-RtPc1 could not be amplified in five isolates. The accumulation of mutations in several elements most likely explains why we failed to amplify these elements by PCR in some isolates. We could not establish any correlation between the presence of the elements and the origin of the isolate. However, the wide distribution of transposons in most Paracoccidioides isolates indicates that they may have been present in the common ancestor before the speciation event.

TEs can be inserted into various regions of the eukaryotic genome, including the flanking regions of euchromatic genes. There are several cases of TE insertions acting in genes regulatory pathways, which can interfere with their activation or inactivation [4,33-35], suffering higher selective pressures. Hence, the high similarity observed between all RtPc 1, RtPc3 and RtPc5 elements suggests that the elements described in the present work are still active or under purifying selection, otherwise several mutations would be expected. Through phylogenetic analysis, we can infer that the two copies of the element LTR-Gypsy-RtPc1 present in the genome of $P$. brasiliensis (isolate $\mathrm{Pb} 18$ ) group together with elements of the genome of $P$. lutzii (isolate $\mathrm{Pb} 01)$. The retrotransposition mechanism of LTR, "copy and paste", is equivalent to gene duplication; hence, the emergence of specific RtPC1 lineages in Pb01 and Pb18 isolates could occur concomitantly with the RtPc1 copies present in the common ancestor. If this process occurred independently in P. brasiliensis and P. lutzii, we should see a distribution similar to that shown in the phylogenetic trees obtained in the present work (Figure 6). Fungal genomes exhibit a low rate of evolution [36], which could explain why even the specific lineages are still very similar to the non-specific elements. Then, the emergence of $P$. brasiliensis RtPc1-specific lineages due to the accumulation of mutations can occur concurrently with the maintenance of copies of RtPc1 similar to those present in the common ancestor of P. lutzii and P. brasiliensis [37]. However, for the LINE element RtPc5 it was possible to observe a species-specific grouping, although only a single copy of RtPc5 from $P$. brasiliensis (isolate $\mathrm{Pb} 03$ ) has been analyzed. Therefore, we suggest that the RtPc elements were present in the ancestral lineage shared by $P$. lutzii ( $\mathrm{Pb} 01)$ and $P$. brasiliensis ( $\mathrm{Pb} 03$ and $\mathrm{Pb} 18)$ and that the inclusion of these elements must have occurred at this point, especially for LTR-Gypsy- RtPc1 and LINE-Tad-RtPc5. The abundant number and high degree of similarity of these elements indicate that they have undergone a recent amplification process within the genomes of these species. This observation is consistent with the life-cycle of a transposable element [38].

TEs may have two main effects on the host genome: insertion into a new chromosomal locus when the element transposes and ectopic recombination. Two copies of the same element in different chromosomal locations may act as sites for homologous recombination, the effects of which can range from small inversions to major chromosomal rearrangements, such as duplications, deletions or translocations [39,40]. A significant percentage of the Paracoccidioides genome consists of transposable elements that could serve as sites for homologous recombination. In organisms without sexual reproduction, homologous recombination between TEs might be associated with the generation of genetic diversity [11]. Recent evidence suggests that Paracoccidioides species have the potential to undergo sexual reproduction, although to date a sexual reproduction stage has not been described [41,42]. We identified potentially active retrotransposons in Paracoccidioides genomes and also described potentially active DNA transposons. Recently, Shankar and coworkers [43] reported a high expression of retrotransposons in P. lutzii $\mathrm{Pb} 01$ during the dimorphic transition and were able to suppress the transition when the fungi were treated with $17 \beta$-estradiol. Paracoccidioides dimorphism can be triggered by $37^{\circ} \mathrm{C}$ temperatures in hosts or in culture, and the morphological differentiation that takes place during the mycelial-to-yeast transition is considered to be vital to the pathogenesis of PCM $[43,44]$. The transposition of active elements can have major mutational effects. TE insertion near or within a gene may alter or destroy the activity of the gene in several ways, ranging from total inactivation to changes in expression levels or alternative splicing [7]. A recently conducted study in Schizosaccharomyces pombe showed that the transcription of the LTR retrotransposon Tf1 was induced by heat treatment, and genes that were induced by heat could be activated by Tf1 integration. The authors speculate that Tf1 integration has the potential to 


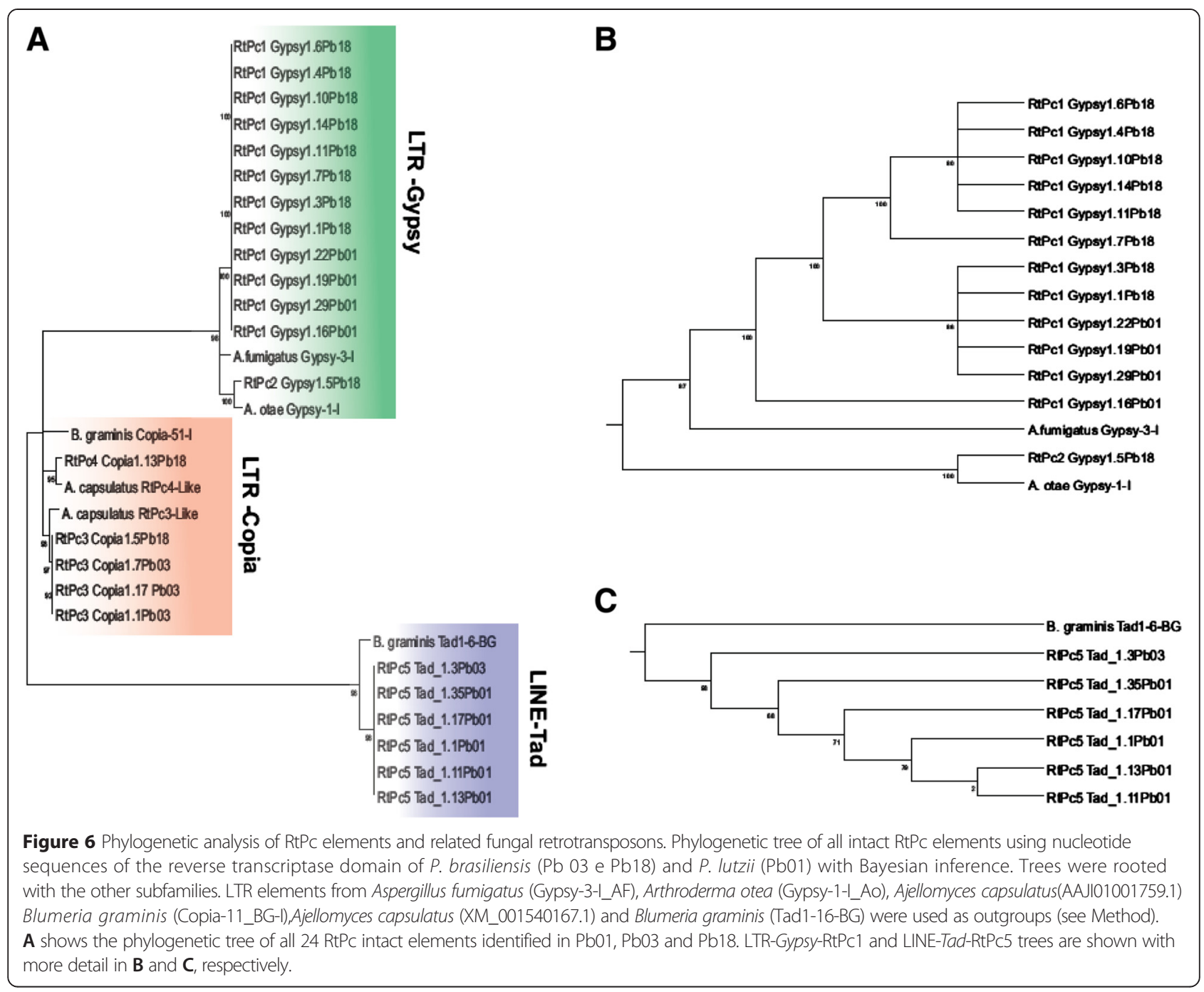

improve the survival of individual cells exposed to environmental stress [45].

\section{Conclusions}

A combined computational and experimental approach was effective in identifying and characterizing five new potentially active retrotransposons in the genomic assemblies of the Paracoccidioides species complex. All elements have been found in both $P$. lutzii and $P$. brasiliensis (S1, PS2 and PS3). This finding suggests that the RtPc elements would have already been present in a common ancestor. Full-length, potentially functional autonomous elements were characterized, and transcripts of all five elements have been detected. Considering the growing body of evidence in the literature indicating the major importance of TEs, it would be interesting to investigate the impact of active retrotransposons on the biology of these dimorphic pathogenic fungi.

\section{Methods}

Bioinformatics and data analysis

Paracoccidioides brasiliensis expressed sequence tags (ESTs) were retrieved from the NCBI dbEST database (http:// www.ncbi.nlm.nih.gov/genbank/dbest/ NCBI EST database release 110111) (Figure 1). The search resulted in 41,558 EST sequences that were stored in a local database. The clustering process involved an initial step employing a reciprocal blast (all versus all blastn) of EST sequences followed by an assembly using the CAP3 program [46]. ESTs were assembled into clusters of overlapping sequences at least 100 bp with $80 \%$ identity. The resulting contigs and singlets, hereafter referred to as clusters, were used to identify regions with similarity to retrotransposons. The blastx and tblastx algorithms [47] were used to compare these groups with sequences from three sequence databases: a) NR, the NCBI non-redundant protein database; b) Repbase, the GIRI (Genetic information Research Institute) database 
of repetitive elements (http://www.girinst.org/repbase/ index.html). The analyses were performed using default parameter values and an E-value of $10^{-6}$. All of the results collected after the various analyses performed in the characterization of each transposon family were stored in a hyperlinked Excel spreadsheet. Each line of the spreadsheet resents a cluster containing a variable number of sequences containing the follow descriptions: query id; seq size; annotation term; e-value and raw score; \% identity; and \% match length (Data not shown). A specific lexical search using eight keywords (retrotransposon, retroviruses, reverse transcriptase, gag, endonuclease, RNase $\mathrm{H}$, integrase, Copia-like) was conducted. To get a start on clusters for the queries against Paracoccidioides genomes, the EST clusters common to both the NR and specific databases were identified (Figure 1).

\section{Databases}

The following databases and versions were used: a) the $P$. brasiliensis genomic sequences (isolates $\mathrm{Pb} 01, \mathrm{~Pb} 03$ and Pb18) from the Paracoccidioides brasiliensis Sequencing project, Broad Institute of MIT and Harvard (release 12-2010 (http://www.broadinstitute.org/annotation/genome/ paracoccidioides_brasiliensis/Downloads.html); b) the NR protein database from NCBI (release 10.2011-186); c) TEfam (release VB2010-12); and d) Repbase (release RepBase16.11).

\section{Annotation and classification of sequences}

Structural and functional annotations were performed using Artemis: Genome Browser and Annotation Tool (http://www.sanger.ac.uk/Software/Artemis/) and PERL scripts developed to analyze and parse the results. Retrotransposons were manually annotated based on the similarities of the sequences to proteins stored in the SeqDBLite fraction of the Gene Ontology database (http://www.geneontology.org), Pfam (Protein Families - http://pfam.sanger.ac.uk/), CDD (Conserved Domain Database www.ncbi.nlm.nhi.gov/Structure/cdd/wrpsb.cgi) and files used by InterProScan, as well as based on the presence of specific signatures, such as long terminal repeats - LTRs. The LTR regions, which are specific features of retrotransposons, were identified by the Tandem Repeat Finder (TRF) [48] and LTR_FINDER programs [49]. Functional annotation was confirmed by manual inspection. Classification of retrotransposons was based on nomenclature previously established for classifying TEs [2,24]. Retrotransposons were classified by class, subclass, order and superfamily. In this report, two retrotransposons were considered to belong to the same family if they shared $80 \%$ (or more) sequence similarity in at least $80 \%$ of the aligned sequences in either their coding region or internal domain or within their long terminal repeats in segments of at least $80 \mathrm{bp}$ (80-80-80 rule) [2]. The RTclass1 computational tool was used to detail the classification of non-LTR retrotransposon elements (http://www.girinst.org/RTphylogeny/RTclass1/) [24]. All consensus sequences for each putative element were submitted to Repbase and compared using the Repeat Masking algorithm to identify related elements (http://www.girinst.org/censor/index.php).

\section{Phylogenetics analysis and evolution of RtPc elements}

Regarding the phylogenetic inference, to standardize and avoid different substitution rates among the sequences, only the Reverse Transcriptase (RT) domains from complete elements were considered. Individual alignments were created for each RtPc family sequence using Muscle 4.04 [50], and the results were manually inspected. Each consensus sequence was used in sequence similarity searches using BLAST [47] against nr/nt NCBI databases to identify the closest related fungal retrotransposon. Additionally, Repbase was used to identify putative orthologous sequences. The best evolutionary model was predicted by jModelTest [51], and the Bayesian inference (BI) was evaluated with BEAST 2.1.3 [52]. During the BI analysis process, the following parameters were adopted: a) GTR $+\mathrm{I}+\Gamma$ model (a four-category gamma distribution) and b) analyses were performed for $100,000,000$ generations with a sampling frequency of 100 and with a burnin of 2,500,000. Divergence time dates were used as priors when present in uTimetree.org [53] with the following parameters: a) a Calibrated Yule tree structure and b) a lognormal strict clock with nucleotide substitution rate $6.3 \times 10-$ 3 bp/Myr [36]. Aspergillus fumigatus (Repbase:Gypsy3-I_AF), Arthroderma otea (Repbase:Gypsy-1-I_Ao), Ajellomyces capsulatus (Genbank:AAJI01001759.1) Blumeria graminis (Repbase:Copia-11_BG-I), Ajellomyces capsulatus (Genbank:XM_001540167.1) and Blumeria graminis (Repbase:Tad1-16-BG) sequences were used as outgroups for RtPc 1, 2, 3, 4 and 5, respectively.

\section{P. brasiliensis isolates and growth conditions}

The Paracoccidioides isolates are listed in Table 3. Fungal isolates were maintained by periodic subculturing using YPD medium (5 g.L $\mathrm{L}^{-1}$ yeast extract, 10 g.L $\mathrm{L}^{-1}$ bactopeptone, 15 g. $\mathrm{L}^{-1}$ dextrose and 15 g. $\mathrm{L}^{-1}$ agar, $\mathrm{pH} 6.3$ ) at $35^{\circ} \mathrm{C}-37^{\circ} \mathrm{C}$ [54]. To obtain cells in the exponential phase [55], yeast cells were subcultured three times in YPD medium at 5-d intervals. The entire growth of two culture slants was inoculated into Erlenmeyer flasks containing $50 \mathrm{~mL}$ of YPD broth, placed on a reciprocating shaker at $120 \mathrm{rpm}$ and grown for $5-7 \mathrm{~d}$ at $35^{\circ} \mathrm{C}$. The cells were washed with sterile Milli-Q water and frozen in liquid nitrogen prior to DNA extraction.

\section{DNA/RNA extraction}

Total DNA was extracted from the yeast culture following previously described protocols involving maceration of frozen cells [56] with minor modifications. Total RNA 
was extracted from the yeasts cultures as previously reported [57].

\section{Genomic DNA PCR analysis}

PCR reactions were performed on $10 \mathrm{ng}$ of DNA in a $20-\mu \mathrm{L}$ reaction mixture containing $1.5 \mathrm{mM} \mathrm{MgCl}_{2}(50 \mathrm{mM})$, $100 \mathrm{mM}$ of each dNTP, $100 \mathrm{pmol}$ of each oligonucleotide and 1 unit of Taq DNA Polymerase (Promega). Each PCR was performed in a thermocycler (Eppendorf) for 35 cycles. The cycling conditions were as follows: denaturing at $94^{\circ} \mathrm{C}$ for $1 \mathrm{~min}$, annealing at the Tm for each primer for $30 \mathrm{~s}$, and extension at $72^{\circ} \mathrm{C}$ for $1 \mathrm{~min}$. At the end of the $35^{\text {th }}$ cycle, the heat-denaturing step was omitted and extension was allowed to proceed at $72^{\circ} \mathrm{C}$ for $5 \mathrm{~min}$. The primers corresponding to each retrotransposon are listed in Additional file 6.

\section{Cloning and sequencing}

Fragments of approximately $300 \mathrm{bp}$ in length related to the reverse transcriptase of each retrotransposon were amplified by PCR, and the amplicons were cloned into the pGEM-T Easy vector (Promega) and transformed into Escherichia coli DH5 competent cells. The nucleotide sequences of the DNA clones were determined by the dideoxynucleotide chain termination method, using the BigDye 3.1 Kit (Applied Biosystems).

\section{Blotting analysis}

For Southern blot analysis, DNA samples digested with different restriction enzymes (EcoR I, BamH I, Hind III, Bgl II, Hinf I, Hinc II, Acc I, Nco I, Sal I and EcoR V) were separated by electrophoresis on $0.8 \%$ agarose gels and stained with ethidium bromide $(0.5 \mu \mathrm{g} / \mathrm{mL})$. The agarose gels were incubated with $0.25 \mathrm{M} \mathrm{HCl}$ for $30 \mathrm{~min}$, denatured with $0.5 \mathrm{M} \mathrm{NaOH} / 1.5 \mathrm{M} \mathrm{NaCl}$ for $30 \mathrm{~min}$, neutralized with $1 \mathrm{M}$ Tris-base $/ 0.5 \mathrm{M} \mathrm{NaCl}$ for $30 \mathrm{~min}$ and transferred onto nylon membranes in $20 \times \mathrm{SSC}(0.15 \mathrm{M} \mathrm{NaCl} / 0.015 \mathrm{M}$ sodium citrate) for three hours. The membranes were prehybridized (1\% BSA, $500 \mathrm{mM} \mathrm{NaH}{ }_{2} \mathrm{PO}_{4}, 1 \mathrm{mM}$ EDTA and 7\% SDS) [58] for $2 \mathrm{~h}$ at $48^{\circ} \mathrm{C}$ and then hybridized overnight at the same temperature with a ${ }^{32} \mathrm{P}$-labeled probe. The probes used in the hybridization experiments were derived from the internal region of the reverse transcriptase segment of each element. Following hybridization, the membranes were washed four times in $2 \times$ SSC containing $0.1 \%$ SDS at room temperature before being exposed to X-ray film.

For chromoblot analysis, the separation of chromosomesized P. brasiliensis DNA molecules by PFGE (pulsed field gel electrophoresis) was performed as described by Feitosa et al. [54]. The agarose gels were incubated with $0.25 \mathrm{M}$ $\mathrm{HCl}$ for $30 \mathrm{~min}$, denatured with $0.5 \mathrm{M} \mathrm{NaOH} / 1 \mathrm{M} \mathrm{NaCl}$ for $30 \mathrm{~min}$, neutralized with $1 \mathrm{M}$ Tris-base $/ 0.5 \mathrm{M} \mathrm{NaCl}$ for $20 \mathrm{~min}$ and transferred onto nylon membranes in $20 \times$ SSC (0.15 M NaCl/0.015 M sodium citrate). The membranes were prehybridized in a solution containing $50 \%$ formamide,
$5 \times \mathrm{SSC}, 0.5 \%$ Denhardt's solution, $0.1 \mathrm{mg} / \mathrm{mL}$ salmon sperm DNA and $0.1 \mathrm{mg} / \mathrm{mL}$ tRNA at $42^{\circ} \mathrm{C}$ for $1 \mathrm{~h}$ and then hybridized overnight at the same temperature with a ${ }^{32} \mathrm{P}$-labeled probe. The probes used in the hybridization experiments were derived from the internal region of each element, including the ORF. Following hybridization, the membranes were washed three times (30 min each) in $2 \times$ SSC containing $0.1 \%$ SDS at $42^{\circ} \mathrm{C}, 1 \times \mathrm{SSC}$ containing $0.1 \%$ SDS and $0.1 \times \mathrm{SSC}$ containing $0.1 \%$ SDS at $56^{\circ} \mathrm{C}$ before being exposed to X-ray film.

\section{Transcriptional analysis (RT-PCR)}

For RT-PCR, reverse transcription reactions were performed with $2 \mu \mathrm{g}$ of total RNA. cDNA strands were prepared using oligo-dT primers and the standard protocol of Improm II reverse transcriptase (Promega). After cDNA synthesis, PCR reactions were performed with $1 \mu \mathrm{L}$ of cDNA in a $20-\mu \mathrm{L}$ reaction mixture containing $1.5 \mathrm{mM}$ $\mathrm{MgCl}_{2}(50 \mathrm{mM}), 100 \mathrm{mM}$ of each dNTP, 100 pmol of oligonucleotides from the reverse transcriptase region and 1 unit of Taq Platinum DNA Polymerase (Invitrogen). Amplification was performed for 30 cycles. Cycling conditions were as follows: denaturing at $94^{\circ} \mathrm{C}$ for $1 \mathrm{~min}$, annealing at $50^{\circ} \mathrm{C}$ for $30 \mathrm{~s}$ and extension at $72^{\circ} \mathrm{C}$ for $1 \mathrm{~min} . \beta$-tubulin was used as an internal control.

\section{Additional files}

Additional file 1: Structure and organization of RtPc elements of
the Paracoccidioides complex. Schematic representations of RtPC
complete elements are shown. The LTRs are represented by orange
arrows and PBS/PPT by black arrows. The domains are represented as
follows: zinc finger - gray; protease - green; reverse transcriptase -
yellow; RNase H - red; integrase - light blue; chromodomain - dark-blue;
and endonuclease - pink. The ORF with its respective size is represented
above each element. Arrows below the schematic representation indicate
EST groups anchored to the element. The figures are not to scale.
Additional file 2: Consensus sequences of RtPc elements.
Consensus nucleotide sequences of the RtPc elements identified.
Additional file 3: Characteristics of RtPc elements identified in
Paracoccidioides genomes.
Additional file 4: List of all RtPc insertions in Paracoccidioides
genomes. The complete list of all RtPc insertions, indicating the position
of each element in the genomes of Paracoccidioides lutzii (Pb01) and
Paracoccidioides brasiliensis (Pb03 and Pb18).
Additional file 5: Estimation of the insertion time of LTR
retrotransposon elements in the Paracoccidioides lutzii and
Paracoccidioides brasiliensis genomes.
Additional file 6: Primer sequences used to amplify RtPc elements
by PCR and RT-PCR. Table with the sequences of the primers used to
amplify RtPc elements by PCR and RT-PCR.

Competing interests

The authors declare that they have no competing interests.

Authors' contributions

MAS and RACA: designed and performed the experiments, performed bioinformatics analysis and data analysis. MMM: data and bioinformatics analysis and manuscript preparation. LMO: participated in the design and performed the bioinformatics analysis and manuscript preparation. LGL 
performed the phylogenetics analysis. VSA: designed and performed the experiments and manuscript preparation. MSSF, MB and CMAS were the principal investigators for the transcriptome and genome projects of $P$. brasiliensis and provided valuable advice and discussion. JFS contributed with valuable discussion, supervised and participated in the interpretation and manuscript preparation. JCR designed and supervised the bioinformatics analysis, critically evaluated the manuscript and contributed valuable discussion. PSC designed, coordinated, supervised and participated in the interpretation, discussion of results and manuscript preparation. All authors read and approved the final version of the manuscript.

\section{Acknowledgements}

This work was supported by grants from FAPESP to JFS and from FAPEMIG, CAPES and CNPq (Brazil) to PSC. JCR was support by the following grants: a) FAPEMIG APQ-01661-13 and b) CNPq 301652/2012-0 and 486618/2013-7. We would like to thank Dr. José Marcos Ribeiro, Chief of the Vector Biology Section from the Laboratory of Malaria and Vector Research, NIAID for the support.

\section{Author details}

${ }^{1}$ Departamento de Microbiologia, Instituto de Ciências Biológicas, Universidade Federal de Minas Gerais, 31270-901 Belo Horizonte, MG, Brazil. ${ }^{2}$ Programa de Pós-graduação em Bioinformática, Instituto de Ciências Biológicas, Universidade Federal de Minas Gerais, 31270-901 Belo Horizonte, MG, Brazil. ${ }^{3}$ Departamento de Microbiologia, Imunologia e Parasitologia, Escola Paulista de Medicina, Universidade Federal de São Paulo, 04023-062 São Paulo, SP, Brazil. ${ }^{4}$ Laboratório de Biologia Molecular, Instituto de Ciências Biológicas, Universidade de Brasília, 70910-900 Brasília, DF, Brazil. ${ }^{5}$ Laboratório de Biologia Molecular, Instituto de Ciências Biológicas, Universidade Federal de Goiás, 74001-970 Goiânia, GO, Brazil. ${ }^{6}$ Grupo Informática de Biossistemas, Centro de Pesquisas René Rachou, FIOCRUZ-Minas, 30190-002 Belo Horizonte, MG, Brazil. ${ }^{7}$ Departamento de Biologia Geral, Universidade Federal de Minas Gerais, Belo Horizonte, MG, Brazil.

\section{Received: 11 December 2014 Accepted: 23 April 2015}

Published online: 12 May 2015

\section{References}

1. Kazazian HH. Mobile elements: drivers of genome evolution. Science. 2004;303:1626-32.

2. Wicker T, Sabot F, Hua-Van A, Bennetzen JL, Capy P, Chalhoub B, et al. A unified classification system for eukaryotic transposable elements. Nat Rev Genet. 2007:8:973-82.

3. Daboussi M-J, Capy P. Transposable elements in filamentous fungi. Annu Rev Microbiol. 2003;57:275-99.

4. Hua-Van A, Le Rouzic A, Maisonhaute C, Capy P. Abundance, distribution and dynamics of retrotransposable elements and transposons: similarities and differences. Cytogenet Genome Res. 2005;110:426-40.

5. Finnegan DJ. Eukaryotic transposable elements and genome evolution. Trends Genet. 1989;5:103-7.

6. Kapitonov W, Jurka J. A universal classification of eukaryotic transposable elements implemented in Repbase. Nat Rev Genet. 2008:9:411-2. author reply 414.

7. Hua-Van A, Le Rouzic A, Boutin TS, Filée J, Capy P. The struggle for life of the genome's selfish architects. Biol Direct. 2011;6:19.

8. Thon MR, Pan H, Diener S, Papalas J, Taro A, Mitchell TK, et al. The role of transposable element clusters in genome evolution and loss of synteny in the rice blast fungus Magnaporthe oryzae. Genome Biol. 2006;7:R16.

9. Davière JM, Langin T, Daboussi MJ. Potential role of transposable elements in the rapid reorganization of the Fusarium oxysporum genome. Fungal Genet Biol. 2001;34:177-92.

10. Braumann I, van den Berg MA, Kempken F. Strain-specific retrotransposonmediated recombination in commercially used Aspergillus niger strain. Mol Genet Genomics. 2008:280:319-25.

11. De Jonge $R$, Bolton MD, Kombrink A, van den Berg GCM, Yadeta KA, Thomma BPHJ. Extensive chromosomal reshuffling drives evolution of virulence in an asexual pathogen. Genome Res. 2013;23:1271-82.

12. Brummer E, Castaneda E, Restrepo A. Paracoccidioidomycosis: an update. Clin Microbiol Rev. 1993:6:89-117.
13. Prado M, Silva MB, Laurenti $R$, Travassos LR, Taborda CP. Mortality due to systemic mycoses as a primary cause of death or in association with AIDS in Brazil: a review from 1996 to 2006. Mem Inst Oswaldo Cruz. 2009;104:513-21.

14. Franco M. Host-parasite relationships in paracoccidioidomycosis. J Med Vet Mycol. 1987;25:5-18.

15. Matute DR, McEwen JG, Puccia R, Montes BA, San-Blas G, Bagagli E, et al Cryptic speciation and recombination in the fungus Paracoccidioides brasiliensis as revealed by gene genealogies. Mol Biol Evol. 2006;23:65-73.

16. Matute DR, Sepulveda VE, Quesada LM, Goldman GH, Taylor JW, Restrepo A, et al. Microsatellite analysis of three phylogenetic species of Paracoccidioides brasiliensis. J Clin Microbiol. 2006;44:2153-7.

17. Teixeira MM, Theodoro RC, de Carvalho MJA, Fernandes L, Paes HC, Hahn $\mathrm{RC}$, et al. Phylogenetic analysis reveals a high level of speciation in the Paracoccidioides genus. Mol Phylogenet Evol. 2009;52:273-83.

18. Carrero LL, Niño-Vega G, Teixeira MM, Carvalho MJ, Soares CM, Pereira M, et al. New Paracoccidioides brasiliensis isolate reveals unexpected genomic variability in this human pathogen. Fungal Genet Biol. 2008;45:605-12.

19. Carvalho KC, Ganiko L, Batista WL, Morais FV, Marques ER, Goldman GH, et al. Virulence of Paracoccidioides brasiliensis and gp43 expression in isolates bearing known PbGP43 genotype. Microbes Infect. 2005;7:55-65.

20. Teixeira MM, Theodoro RC, Nino-Vega G, Bagagli E, Felipe MSS. Paracoccidioides species complex: ecology, phylogeny, sexual reproduction, and virulence. PLoS Pathog. 2014;10, e1004397.

21. Desjardins CA, Champion MD, Holder JW, Muszewska A, Goldberg J, Bailão AM, et al. Comparative genomic analysis of human fungal pathogens causing paracoccidioidomycosis. PLoS Genet. 2011;7, e1002345.

22. Marini MM, Zanforlin T, Santos PC, Barros RRM, Guerra ACP, Puccia R, et al. Identification and characterization of TC1/mariner-like DNA transposons in genomes of the pathogenic fungi of the Paracoccidioides species complex. BMC Genomics. 2010;11:130.

23. Kidwell MG. Transposable elements and the evolution of genome size in eukaryotes. Genetica. 2002;115:49-63.

24. Kapitonov W, Tempel S, Jurka J. Simple and fast classification of nonLTR retrotransposons based on phylogeny of their RT domain protein sequences. Gene. 2009;448:207-13.

25. Novikova O, Fet $\mathrm{V}$, Blinov A. Non-LTR retrotransposons in fungi. Funct Integr Genomics. 2009;9:27-42.

26. Kijima TE, Innan $\mathrm{H}$. On the estimation of the insertion time of LTR retrotransposable elements. Mol Biol Evol. 2010;27:896-904.

27. Muszewska A, Hoffman-Sommer M, Grynberg M. LTR retrotransposons in fungi. PLoS One. 2011;6, e29425.

28. Galagan JE, Selker EU. RIP: the evolutionary cost of genome defense. Trends Genet. 2004;20:417-23.

29. Galagan JE, Henn MR, Ma L-J, Cuomo CA, Birren B. Genomics of the fungal kingdom: insights into eukaryotic biology. Genome Res. 2005;15:1620-31.

30. Hood ME, Katawczik M, Giraud T. Repeat-induced point mutation and the population structure of transposable elements in Microbotryum violaceum. Genetics. 2005;170:1081-9.

31. Cambareri EB, Jensen BC, Schabtach E, Selker EU. Repeat-induced G-C to A-T mutations in Neurospora. Science. 1989;244:1571-5.

32. Tubio JMC, Tojo M, Bassaganyas L, Escaramis G, Sharakhov IV, Sharakhova MV, et al. Evolutionary dynamics of the Ty3/gypsy LTR retrotransposons in the genome of Anopheles gambiae. PLoS One. 2011;6, e16328.

33. Daboussi MJ. Fungal transposable elements and genome evolution. Genetica. 1997;100:253-60

34. Navarro-Quezada A, Schoen DJ. Sequence evolution and copy number of Ty1-copia retrotransposons in diverse plant genomes. Proc Natl Acad Sci U S A. 2002:99:268-73.

35. Baucom RS, Estill JC, Leebens-Mack J, Bennetzen JL. Natural selection on gene function drives the evolution of LTR retrotransposon families in the rice genome. Genome Res. 2009;19:243-54.

36. Kasuga T, White TJ, Taylor JW. Estimation of nucleotide substitution rates in Eurotiomycete fungi. Mol Biol Evol. 2002;19:2318-24.

37. Böhne A, Brunet F, Galiana-Arnoux D, Schultheis C, Volff J-N. Transposable elements as drivers of genomic and biological diversity in vertebrates. Chromosome Res. 2008;16:203-15.

38. Kidwell M, Lisch D. Transposable elements and host genome evolution. Trends Ecol Evol. 2000;15:95-9.

39. Zhang J, Yu C, Pulletikurti V, Lamb J, Danilova T, Weber DF, et al. Alternative Ac/Ds transposition induces major chromosomal rearrangements in maize. Genes Dev. 2009;23:755-65. 
40. Delprat A, Negre B, Puig M, Ruiz A. The transposon Galileo generates natural chromosomal inversions in Drosophila by ectopic recombination. PLoS One. 2009;4, e7883.

41. Gomes-Rezende JA, Gomes-Alves AG, Menino JF, Coelho MA, Ludovico $P$, Gonçalves $P$, et al. Functionality of the Paracoccidioides mating a-pheromone-receptor system. PLoS One. 2012;7, e47033.

42. Teixeira Mde M, Theodoro RC, Derengowski Lda S, Nicola AM, Bagagli E, Felipe MS. Molecular and morphological data support the existence of a sexual cycle in species of the genus Paracoccidioides. Eukaryot Cell. 2013;12:380-9.

43. Shankar J, Wu TD, Clemons KV, Monteiro JP, Mirels LF, Stevens DA. Influence of $17 \beta$-estradiol on gene expression of Paracoccidioides during mycelia-to-yeast transition. PLoS One. 2011:6, e28402.

44. Carbonell LM, Rodriguez J. Transformation of mycelial and yeast forms of paracoccidioides brasiliensis in cultures and in experimental inoculations. J Bacteriol. 1965;90:504-10.

45. Feng $G$, Leem Y-E, Levin HL. Transposon integration enhances expression of stress response genes. Nucleic Acids Res. 2013;41:775-89.

46. Huang X, Madan A. CAP3: A DNA sequence assembly program. Genome Res. 1999:9:868-77.

47. Altschul SF, Gish W, Miller W, Myers EW, Lipman DJ. Basic local alignment search tool. J Mol Biol. 1990:215:403-10.

48. Benson G. Tandem repeats finder: a program to analyze DNA sequences. Nucleic Acids Res. 1999:27:573-80

49. Xu Z, Wang H. LTR_FINDER: an efficient tool for the prediction of full-length LTR retrotransposons. Nucleic Acids Res. 2007:35(Web Server issue):W265-8.

50. Edgar RC. MUSCLE: multiple sequence alignment with high accuracy and high throughput. Nucleic Acids Res. 2004;32:1792-7.

51. Posada D. jModelTest: phylogenetic model averaging. Mol Biol Evol. 2008;25:1253-6.

52. Bouckaert R, Heled J, Kühnert D, Vaughan T, Wu C-H, Xie D, et al. BEAST 2: a software platform for Bayesian evolutionary analysis. PLOS Comput Biol. 2014;10, e1003537.

53. Hedges SB, Dudley J, Kumar S. TimeTree: a public knowledge-base of divergence times among organisms. Bioinformatics. 2006;22:2971-2.

54. Feitosa Ldos S, Cisalpino PS, dos Santos MRM, Mortara RA, Barros TF, Morais FV, et al. Chromosomal polymorphism, syntenic relationships, and ploidy in the pathogenic fungus Paracoccidioides brasiliensis. Fungal Genet Biol. 2003:39:60-9.

55. San-Blas G, Suzuki S, Hearn V, Pinel C, Kobayashi H, Mendez C, et al. Fungal polysaccharides. J Med Vet Mycol. 1994;32 Suppl 1:321-8.

56. Girardin HLJ. DNA extraction and quantitation. In: Mol Biol Pathog fungi A Lab Man. 1994. p. 5-9.

57. Batista WL, Barros TF, Goldman GH, Morais FV, Puccia R. Identification of transcription elements in the $5^{\prime}$ intergenic region shared by LON and MDJ heat shock genes from the human pathogen Paracoccidioides brasiliensis. Evaluation of gene expression. Fungal Genet Biol. 2007;44:347-56.

58. Church GM, Gilbert W. Genomic sequencing. Proc Natl Acad Sci U S A. 1984:81:1991-5.

59. Morais FV, Barros TF, Fukada MK, Cisalpino PS, Puccia R. Polymorphism in the gene coding for the immunodominant antigen gp43 from the pathogenic fungus Paracoccidioides brasiliensis. J Clin Microbiol. 2000;38:3960-6.

\section{Submit your next manuscript to BioMed Central and take full advantage of:}

- Convenient online submission

- Thorough peer review

- No space constraints or color figure charges

- Immediate publication on acceptance

- Inclusion in PubMed, CAS, Scopus and Google Scholar

- Research which is freely available for redistribution 